\title{
Epigenetics Theoretical Limits of Synthetic Genomes : the cases of Artificials Caulobacter (C. eth-2.0), Mycoplasma Mycoides (JCVI-Syn 1.0, JCVI-Syn 3.0 and JCVI_3A), E-coli and YEAST chr XII
}

«Solved by standard Gammas, unvarying Deltas, uniform Epsilons. Millions of identical twins. The principle of mass production at last applied to biology. »

in « BRAVE NEW WORLD » Chapter 1 , Aldous Huxley 1931

https://www.huxley.net/bnw/one.html

Jean-claude Perez, Retired interdisciplinary researcher (IBM European Research Center on Artificial Intelligence), 7 avenue de terre-rouge F33127 Martignas Bordeaux metropole France. email: jeanclaudeperez2@gmail.com, more on :

http://novelnewbiologyresearch.blogspot.com/2018/01/jean-claude-perez-gabriella-loza-and.html

\begin{abstract}
:
In (Venetz et al., 2019), authors rebuilt the essential genome of Caulobacter crescentus through the process of chemical synthesis rewriting and studied the genetic information content at the level of its essential genes. Then, they reduced the native Caulobacter crescentus native Caulobacter NA1000 genome sequence real genome ( 4042929 bp ) to the 785,701-bp reduced synthetic genome. Here we demonstrate the existence of a palindromic-like mirror structure that exists in real genomes and disappears totally in the synthetic genome. This biomathematic meta-organization is based on characteristic proportions of Fibonacci numbers between DNA single strand nucleotides proportions TC / AG on the one hand and TG / AC on the other hand. In both cases, we suggest that this meta-structure enhances the three-dimensional cohesion of the two DNA strands of the genome. We then generalize this study to the different synthetic genomes and synthetic cells published by the Craig Venter Institute on Mycoplasma Mycoides JCVI-syn1.0 (in 2010), JCVI-syn3.0 (in 2016) and JCVI-syn3A (in 2019). Finally, in the discussion section, we extend this study to synthetic genomes of E-Coli and Yeast chromosome XII.
\end{abstract}

\section{Key Words :}

Synthetic genomes, Epigenetics, Transposons, Biomathematics, Fibonacci numbers

\section{Introduction :}

The story which led to the development of the first synthetic genome JCVI-syn1.0 has its origins as far back as 1995, when Venter and his team published the sequence of Mycoplasma genitalium (Fraser, 1995) and (Sleator, 2010). In 2010, a 1079-kb genome based on the genome of Mycoplasma mycoides (JCV-syn1.0) was chemically synthesized and supported cell growth when transplanted into cytoplasm. (Gibson, 2010). In 2016, Hutchinson et al design, build, and test cycle to reduce this Mycoplasma mycoides genome to $531 \mathrm{~kb}$ (473 genes). JCV-syn3.0 retains genes involved in key processes such as transcription and translation, but also contains 149 genes of unknown function. Since 2012 the Synthetic Yeast Genome Project (Sc2.0 http://syntheticyeast.org/sc2-0/ ) results from a worldwide partnership, « Sc2.0 International Consortium team », members spanning 4 continents to provide remote mentorship and solve challenges associated with synthetic individual chromosome design features and assembly (Jee Loon Foo 2018). Read the analysis in §Discussion. In January 2019, Breuer et al. published a synthetic cell resulting from the synthetic genome JCVIsyn3A, a robust minimal cell with a $543 \mathrm{kbp}$ genome and 493 genes, providing a versatile platform to study the basics of life. Simultaneously, in 2019, Venetz et al. reduced the native Caulobacter crescentus NA1000 genome sequence real genome ( $4042929 \mathrm{bp}$ ) to the 785,701-bp reduced synthetic genome Caulobacter ethensis-2.0 (C. eth-2.0). Finally, also in 2019 (Fredens, 2019), researchers published a synthetic genome of $E$ COLI changing systematically 
genetic code equivalent codons. They replaced every occurrence of the serine codon TCG with AGC, every TCA (also serine) with AGT, and every TAG (stop) with TAA. Read the analysis in §Discussion.

In a completely different field, 30 years ago, we had just published the first 2 French books on Artificial Intelligence (AI) neural networks (Perez, 1988; Perez, 1989; Perez, 1990a). It is the exploration of our network FRACTAL CHAOS (Perez 1990c) which will reveal a hypersensitivity of this network to successive ratios of Fibonacci numbers, for example 34/21 (Perez, 1990b). While the big project of sequencing of the human genome "HUGO" just begins, we have the intuition to look for ratios of Fibonacci numbers between the contiguous proportions of TCAG nucleotides of genes and small genomes available at that time (like HIV, mtDNA, viruses, bacteria, or small genes). We published a first article in 1991 (Perez, 1991; Marcer, 1992) demonstrating the evidence of such biomathematic structures (Perez, 1991). This discovery was completely published 22 years ago in the book "DNA decrypted" (Perez, 1997). This method, which the Nobel prize winner Luc Montagnier called "DNA supracode" (Fleaux, 1995), was used to search exaustively in DNA searched exhaustively in DNA sequences for remarkable proportions of Fibonacci numbers ( https://en.wikipedia.org/wiki/Fibonacci_number ) between nucleotides called "resonances": for example if a contiguous sequence of 377 bases TCAG is subdivided into $233(C+A)$ and $144(T+G)$, there is a resonance of CA / TG of length 377 (where 144, 233 and 377 are three Fibonacci numbers). In (Perez, 2017a), it is precisely such resonances CA / TG that characterize this optimality of the mtDNA genome of humans. It is still such resonances that are affected during mutations associated with cancers. In particular, we have analyzed this type of resonance in the 3 respective mtDNA genomes of humans, mice, and the famous naked mole rat as well as in more than a dozen other mammalian species.

In a comprehensive analysis of all (ALL) listed mutations of the human mitochondrial mtDNA genome associated with cancers: effectively, multiple mutations associated with the mitochondrial genome of tumor cells have been reported. An open question is whether these mutations are only the CONSEQUENCE of the cancer process or if, on the contrary, they would be a possible ORIGINAL CAUSE of the cancer genesis process. In a paper in preparation (Perez, 2019) we'll propose a generic and universal law (of a numerical nature) allowing us to detect and classify these mutations at the early stage of the genesis of the tumors. Finally, in (Perez 2019) we will present a generic law of prediction and classification of tumors by the simple analysis of the DNA SUPRACODE of the mitochondrial genomes associated with these tumors. In this upcoming article, we analyse all known somatic mutations listed all cancers combined. We then discover a global strategy of mutation of all these basic somatic mutations materialized by a numerical score which systematically increases in ALL the cases of elementary somatic mutations related to 91 referenced cases involved in 9 different cancers (prostate, pancreatic, colon, thyroid, bladder, breast, head $\S$ neck, meduloblastoma, ovarian ) with a success rate of $100 \%$. This predictive method should make it possible to categorize and classify the potential pathogenicity of tumors from the early stage. Particularly, we find an interesting symmetric property of resonances with very short periods: for example, the resonances 3 (1 TC 2AG) and 3 (2TC $1 \mathrm{AG})$ correspond to the symmetrical beginnings of the Fibonacci and Lucas sequences. Similarly, the resonances $5(2 \mathrm{TC}, 3 \mathrm{AG})$ and (3TC $2 \mathrm{AG})$ correspond to the symmetrical beginnings of the Fibonacci and FibLuc ${ }^{1}{ }^{2}$ sequences. By looking for these resonances in all the known tumor

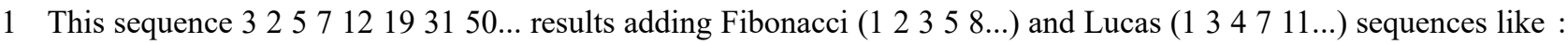
$2(1+1), 5(2+3), 7(3+4), 12(5+7) \ldots$ Curiously, we discovered this sequence in (Perez, 2017b) resulting from stationary waves observed in DUF1220 repeat proteins in mammals brain coding DNA genomes.

2 This sequence was also curiously used in new orleans jazz negro spiritual music (Parayon 2011). 
mutations of human mtDNA genomes applied to the genomes inherited by evolution of the RSRS mother sequence (EVE), it appears the functional role of such local resonances whose repercussion on the global scale of the genome becomes a indicator of early diagnosis of tumors.

It is this type of symmetry that we will generalize in this article by extending it to longer Fibonacci, and Lucas sequences.

Example $34 \mathrm{TCAG}==>13 \mathrm{TC}, 21 \mathrm{AG}$ in one hand (regular) and $34 \mathrm{TCAG}==>21 \mathrm{TC}, 13 \mathrm{AG}$ in other hand (reverse).

\section{Experimental section :}

\section{Part I : Genomes analysed :}

We will analyze 8 bacterial genomes, 3 real reference genomes, one transgenic genome, and four synthetic genomes.

\section{==> The 2 Caulobacter genomes :}

Name : NA1000 real

Reference : Caulobacter crescentus NA1000, complete genome

Publication : Venetz, 2019

Length : 4042929 bp

Access : native Caulobacter NA1000 genome sequence [National Center for Biotechnology Information (NCBI) accession no. $\mathrm{NC}$ 011916.1]

https://www.ncbi.nlm.nih.gov/nuccore/NC 011916.1

Name : Ethensis CETH 2.0

Reference : Synthetic Caulobacter sp. 'ethensis' strain CETH2.0 chromosome, complete genome

Publication ; Venetz, 2019

Length : $785701 \mathrm{bp}$

Access : https://www.ncbi.nlm.nih.gov/nuccore/CP035535

\section{==> The 6 Mycoplasma Mycoides genomes :}

Name : MycRef

Reference : Mycoplasma mycoides subsp. mycoides strain izsam_mm5713, complete genome

Publication : Orsini, 2015

Length : 1192498 bp

Access : https://www.ncbi.nlm.nih.gov/nuccore/CP010267.1?report=genbank

Name : JCVI-syn1.0

Reference : Synthetic Mycoplasma mycoides JCVI-syn1.0 clone sMmYCp235-1, complete sequence

Publication : Gibson, 2010

Length : 1078809 bp

Access : https://www.ncbi.nlm.nih.gov/nuccore/296455217

Name : Capritrans

Reference : Mycoplasma mycoides subsp. capri str. GM12 transgenic clone tetM-lacZ, 
complete genome

Publication : Direct Submission

JOURNAL Submitted (14-MAY-2009) The J. Craig Venter Institute, 9702 Medical Center Drive, Rockville, MD 20850, USA

Length : 1089202 bp

Access : https://www.ncbi.nlm.nih.gov/nuccore/CP001621.1

Name : Capri real

Reference : Mycoplasma mycoides subsp. mycoides SC str. PG1

Length : 1211703 bp

Access : $\underline{\text { https://www.ncbi.nlm.nih.gov/nuccore/NC 005364.2 }}$

Name : JCVI-Syn3.0

Reference : Synthetic bacterium JCVI-Syn3.0, complete genome

Publication : Hutchinson, 2016

Length :531490 bp

Access : https://www.ncbi.nlm.nih.gov/nuccore/CP014940.1

Name : JCVI-Syn3A

Reference : Synthetic bacterium JCVI-Syn3A chromosome, complete genome

Publication : Breuer, 2019

Length : 543379 bp

Access : $\underline{\text { https://www.ncbi.nlm.nih.gov/nuccore/CP016816.2 }}$

\section{Part II : Computing DNA Supra Code Resonances :}

Let us consider the 2 digital sequences :

Fibonacci: 1123581321345589

Lucas: 213471118294776

For any contiguous sequence of nucleotides, one will search for "resonance" or exact proportions of the TG / CA types then mainly TC / AG.

For example, if 34 TCAG bases are subdivided exactly into $13 \mathrm{TC}$ bases and $21 \mathrm{AG}$ bases, we will consider having discovered a TC / AG resonance of length 34. We will do the same for the search for Lucas resonances. The whole genome is explored by taking each of the positions as successive exploration points. On the other hand, the genome being circular, the analysis from the last pivots at the end of the sequence is looped back to the positions of the start nucleotides.

We will thus search for 2 symmetrical types of resonances:

Main resonances (or forward): Exp. 34 TCAG $==>13$ TC, 21 AG.

Inverse Resonances (or backward): Exp. $34 \mathrm{TCAG}==>21 \mathrm{TC}, 13 \mathrm{AG}$.

For each length of Fibonacci (or Lucas) 35813213455 89, we memorize the respective accumulations of the forward resonances on the one hand, and backward on the other hand.

It appears then that these 2 values are very close in the case of REAL genomes, whereas they are very different in the case of SYNTHETIC genomes.

We will therefore consider very significant: The forward / backward ratios. Forward-backward spreads. Since the lengths of real and synthetic genomes are generally very different, we will weight the forward-backward differences by the respective lengths of the real or synthetic genomes. 


\section{Results :}

We analyse here, in one hand, Caulobacter crescentus NA1000 genome and synthetic genome Caulobacter ethensis-2.0 (C. eth-2.0), and, in other hand, Mycoplasma Mycoides JCVI-syn1.0, JCVI-syn3.0 and JCVI-syn3A.

Part I : Caulobacter crescentus NA1000 genome and synthetic genome Caulobacter ethensis2.0 (C. eth-2.0).

The actual NA1000 genome being about 5 times longer than the synthetic genome C. eth-2.0, one might think that the comparison of these 2 genomes is skewed. However, in all the above results, we had already incorporated this difference by weighting the results by the length of the respective genomes.

TC/AG analysis :

Nota : All tables in this article are identical: each box contains 4 numerical values: 1 / The number "L" of Fibonacci or Lucas constituting the length of the sub-sequence analyzed. 2 I The cumulated volume of the corresponding resonances $(n \times L)$ in regular exploration (forward). 3 / The cumulative volume of the corresponding resonances $(L x n)$ in reverse (backward) exploration. 4 / The ratio of the 2 values below regular / reverse.

Table 1 - TC/AG Fibonacci and Lucas analysis for real NA1000 genome and synthetic Caulobacter 2.0.

\begin{tabular}{|c|c|c|c|c|c|c|c|}
\hline \multicolumn{4}{|c|}{ TC/AG Real genome NA1000 } & \multicolumn{4}{|c|}{ TC/AG Synthetic genome CAULOBACTER 2.0} \\
\hline \multicolumn{2}{|c|}{ Fibonacci } & \multicolumn{2}{|c|}{ Lucas } & \multicolumn{2}{|c|}{ Fibonacci } & \multicolumn{2}{|c|}{ Lucas } \\
\hline 3 & $\begin{array}{l}1580254 \\
1582201 \\
0.9987694357\end{array}$ & 3 & $\begin{array}{l}1582201 \\
1580254 \\
1.00123208\end{array}$ & 3 & $\begin{array}{l}290047 \\
293417 \\
0.9885146396\end{array}$ & 3 & $\begin{array}{l}293417 \\
290047 \\
1.011618807\end{array}$ \\
\hline 5 & $\begin{array}{l}1346353 \\
1346512 \\
0.9998819171\end{array}$ & 4 & $\begin{array}{l}993497 \\
994619 \\
0.9988719299\end{array}$ & 5 & $\begin{array}{l}239292 \\
242875 \\
0.9852475553\end{array}$ & 4 & $\begin{array}{l}194318 \\
198624 \\
0.9783208474\end{array}$ \\
\hline 8 & $\begin{array}{l}924521 \\
926003 \\
0.9983995732\end{array}$ & 7 & $\begin{array}{l}1186993 \\
1186953 \\
1.0000337\end{array}$ & 8 & $\begin{array}{l}168546 \\
172325 \\
0.9780705063\end{array}$ & 7 & $\begin{array}{l}209444 \\
211890 \\
0.9884562745\end{array}$ \\
\hline 13 & $\begin{array}{l}650861 \\
652564 \\
0.9973902943\end{array}$ & 11 & $\begin{array}{l}661049 \\
662784 \\
0.9973822542\end{array}$ & 13 & $\begin{array}{l}120565 \\
124586 \\
0.9677251055\end{array}$ & 11 & $\begin{array}{l}123932 \\
128203 \\
0.966685647\end{array}$ \\
\hline 21 & $\begin{array}{l}377329 \\
378580 \\
0.9966955465\end{array}$ & 18 & $\begin{array}{l}489878 \\
492487 \\
0.9947023982\end{array}$ & 21 & $\begin{array}{l}7427877737 \\
0.9555038141\end{array}$ & 18 & $\begin{array}{l}9249996693 \\
0.9566256089\end{array}$ \\
\hline 34 & $\begin{array}{l}188645 \\
190539 \\
0.9900597778\end{array}$ & 29 & $\begin{array}{l}232050 \\
233697 \\
0.9929524127\end{array}$ & 34 & $\begin{array}{l}4081644246 \\
0.9224788681\end{array}$ & 29 & $\begin{array}{l}4889552692 \\
0.9279397252\end{array}$ \\
\hline 55 & $\begin{array}{l}71250 \quad 72070 \\
0.9886221729\end{array}$ & 47 & $\begin{array}{l}104152 \\
104668 \\
0.9950701265\end{array}$ & 55 & $\begin{array}{l}1813120974 \\
0.8644512253\end{array}$ & 47 & $\begin{array}{l}24743 \quad 27988 \\
0.8840574532\end{array}$ \\
\hline 89 & $\begin{array}{l}1969419535 \\
1.008139237\end{array}$ & 76 & $\begin{array}{l}3080431161 \\
0.9885433715\end{array}$ & 89 & $\begin{array}{l}6300 \quad 8172 \\
0.7709251101\end{array}$ & 76 & $\begin{array}{ll}9026 & 11226 \\
0.8040263674\end{array}$ \\
\hline
\end{tabular}


Caulobacter TCIAG Fibonacci Resonances

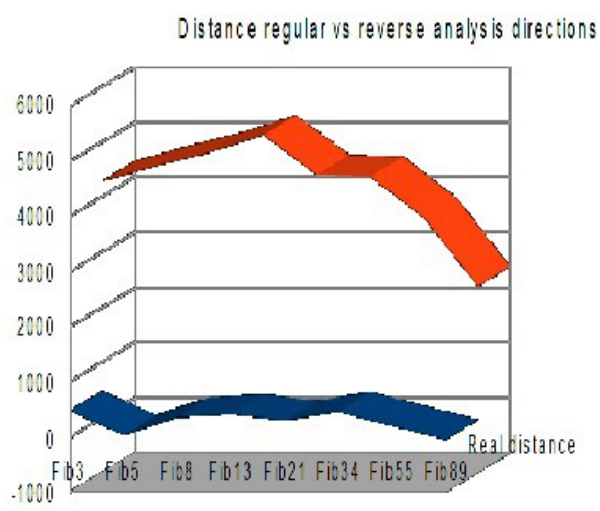

Caulobacter TCIRG Lucas resonances

Dis tance regular (forw ard) vs reverse (backward) analys is directions

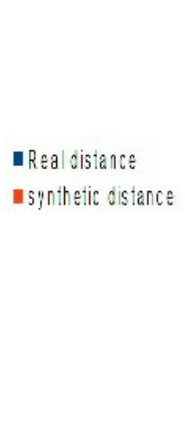

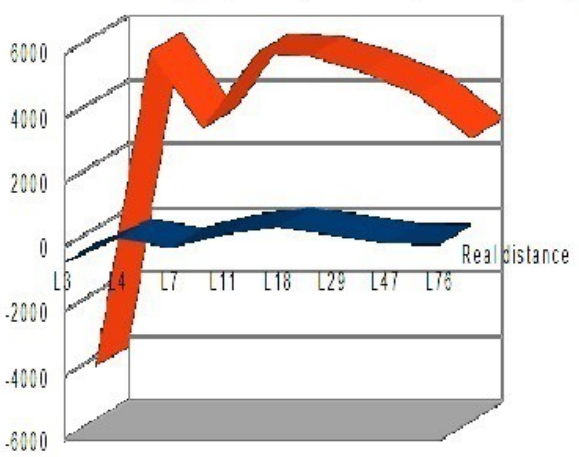

- Real distance - 5 ynthetic distance

Figure 1 - Comparing TC/AG Fibonacci and Lucas distances in real and synthetic Caulobacter genomes ( regularreverse distances weighted by the length of the genome, see detailed data in suplementary materials ).

example of weighting by the length of the genome computing here the case of synthetic caulobacterium genome (case of the first Fibonacci resonance of length $=3$ nucleotides) :

regular - reverse distance $=290047-293417=-3370$

weighting by the genome length : ${ }^{-} 3370 \div 785750=-0.004288895959$

normalization multiply by $1000000=1000000 \times-0.004288895959={ }^{-} 4288.895959=-4289$

(see more details in suplementary materials page 2).

In the figure on the left, the average ratio of weighted distances by genome length between real genome and synthetic genome is $\mathbf{1 4 . 3 9}$ for TC / AG Fibonacci resonances (see supplementary materials).

For information, the same ratio related to Lucas $\mathrm{TC} / \mathrm{AG}$ is $=\mathbf{1 4 . 3 4 5 4 8 4}$

Computing details :

$\begin{array}{llllllllll}\text { real genome abs. Distances : } & 482 & 40 & 367 & 422 & 310 & 469 & 203 & 39\end{array}$

synthetic genome abs. Distances : $4289 \quad 4560 \quad 4810 \quad 5118 \quad 4403 \quad 4366 \quad 3619 \quad 2383$

cumulating real genome abs. Distances :2332

cumulating synthetic genome abs. Distances : 33548

Ratio synthetic genome abs. Distances / real genome abs. Distances $=14.38593482$

TG/AC analysis :

Table 2 - TG/AC Fibonacci and Lucas analysis for real NA1000 genome and synthetic Caulobacter 2.0.

\begin{tabular}{|c|c|c|c|c|c|c|c|}
\hline \multicolumn{4}{|c|}{ TG/CA Real genome NA1000 } & \multicolumn{4}{|c|}{ TG/CA Synthetic genome CAULOBACTER 2.0} \\
\hline \multicolumn{2}{|c|}{ Fibonacci } & \multicolumn{2}{|c|}{ Lucas } & \multicolumn{2}{|c|}{ Fibonacci } & \multicolumn{2}{|c|}{ Lucas } \\
\hline 3 & $\begin{array}{l}1607779 \\
1614874 \\
0.9956064684\end{array}$ & 3 & $\begin{array}{l}1614874 \\
1607779 \\
1.00441292\end{array}$ & 3 & $\begin{array}{l}296256 \\
299993 \\
0.9875430427\end{array}$ & 3 & $\begin{array}{l}299993 \\
296256 \\
1.012614091\end{array}$ \\
\hline 5 & $\begin{array}{l}1349192 \\
1358378 \\
0.993237523\end{array}$ & 4 & $\begin{array}{l}1011587 \\
1022025 \\
0.9897869426\end{array}$ & 5 & $\begin{array}{l}244860 \\
248873 \\
0.9838753099\end{array}$ & 4 & $\begin{array}{l}195156 \\
199978 \\
0.9758873476\end{array}$ \\
\hline 8 & $\begin{array}{l}915781 \\
925976 \\
0.9889899954\end{array}$ & 7 & $\begin{array}{l}1174192 \\
1180896 \\
0.9943229548\end{array}$ & 8 & $\begin{array}{l}169216 \\
174299 \\
0.9708374689\end{array}$ & 7 & $\begin{array}{l}213326 \\
216126 \\
0.9870445944\end{array}$ \\
\hline
\end{tabular}




\begin{tabular}{|c|c|c|c|c|c|c|c|}
\hline 13 & $\begin{array}{l}642769 \\
652548 \\
0.9850141292\end{array}$ & 11 & $\begin{array}{l}654997 \\
665831 \\
0.9837286038\end{array}$ & \multicolumn{2}{|c|}{$\begin{array}{l}13 \\
120130 \\
125243 \\
959176\end{array}$} & 11 & $\begin{array}{l}123819 \\
128731 \\
0.9618429127\end{array}$ \\
\hline 21 & $\begin{array}{l}384426 \\
392873 \\
0.9784994133\end{array}$ & 18 & $\begin{array}{l}489376 \\
497649 \\
0.9833758332\end{array}$ & 21 & $\begin{array}{l}7390978870 \\
0.9370990237\end{array}$ & 18 & $\begin{array}{l}9267096745 \\
0.9578789602\end{array}$ \\
\hline 34 & $\begin{array}{l}209433 \\
218299 \\
0.9593859798\end{array}$ & 29 & $\begin{array}{l}251102 \\
259033 \\
0.9693822795\end{array}$ & 34 & $\begin{array}{l}42458 \quad 46179 \\
0.9194222482\end{array}$ & 29 & $\begin{array}{l}5014553889 \\
0.9305238546\end{array}$ \\
\hline 55 & $\begin{array}{l}9175697158 \\
0.9443998436\end{array}$ & 47 & $\begin{array}{l}127067 \\
133142 \\
0.9543720239\end{array}$ & 55 & $\begin{array}{ll}20641 & 22427 \\
0.9203638471\end{array}$ & 47 & $\begin{array}{l}2730529582 \\
0.9230275167\end{array}$ \\
\hline 89 & $\begin{array}{l}31478 \quad 34303 \\
0.917645687\end{array}$ & 76 & $\begin{array}{l}45537 \quad 49636 \\
0.9174188089\end{array}$ & 89 & $\begin{array}{ll}8188 & 9531 \\
0.859091386\end{array}$ & 76 & $\begin{array}{l}11334 \quad 12669 \\
0.8946246744\end{array}$ \\
\hline
\end{tabular}

Caulobacter TG/AC Fibonacciresonances

Dis tances regular (iforw ardi vs reverse backw ardi) analys is direction

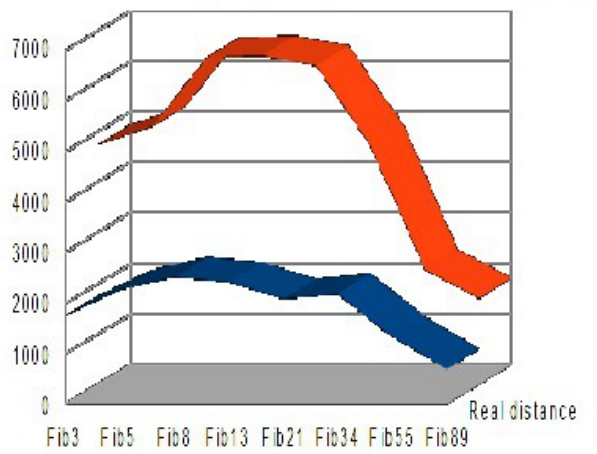

Caulobacter TG/RC Lucas resonaces

Dis tances regular (forvard) vs reverse ibackwardi analys is direction

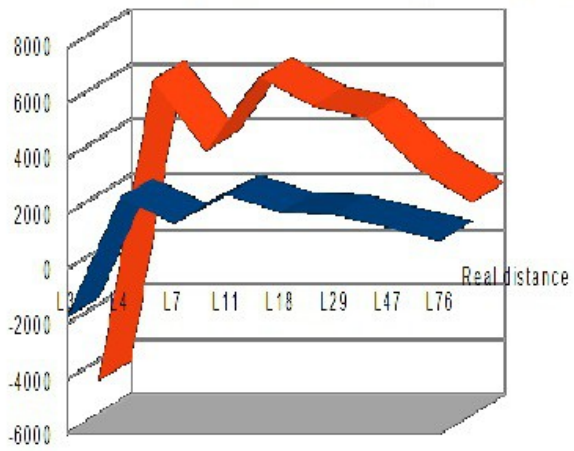

- Real distance II synthetic distance

Figure 2 - Comparing TG/AC Fibonacci and Lucas distances in real and synthetic Caulobacter genomes ( regular-reverse distances weighted by the length of the genome, see detailed data in suplementary materials ).

\section{Part II : Mycoplasma Mycoides JCVI-syn1.0 (2010), JCVI-syn3.0 (2016) and JCVI- syn3A (2019)}

In 2010, a 1079-kb genome based on the genome of Mycoplasma mycoides (JCV-syn1.0) was chemically synthesized and supported cell growth when transplanted into cytoplasm. (Gibson, 2010). In 2016, Hutchinson et al. design, build, and test cycle to reduce this Mycoplasma mycoides genome to $531 \mathrm{~kb}$ (473 genes). JCV-syn3.0 retains genes involved in key processes such as transcription and translation, but also contains 149 genes of unknown function. In the following section, we compare 6 (six) genomes : two reference real strain mycoplasma genomes including CAPRI strain, one transgenic building strain and the 3 strong JCV Labs ; synthetic genomes.

Table 3 - Comparing TC/AG Fibonacci analysis for 6 real or synthetic Mycoplasm genomes. 


\begin{tabular}{|c|c|c|c|c|c|c|c|c|c|c|c|}
\hline \multicolumn{6}{|c|}{ Mycoplasm REF real genomes } & \multicolumn{6}{|c|}{ Synthetic Mycoplasm genomes } \\
\hline \multicolumn{4}{|c|}{ Natural reference genomes } & \multicolumn{2}{|c|}{$\begin{array}{l}\text { Transgenic } \\
\text { genome }\end{array}$} & \multicolumn{6}{|c|}{ Synthetic genomes } \\
\hline \multicolumn{2}{|c|}{$\begin{array}{l}\text { Reference real } \\
\text { strain }\end{array}$} & \multicolumn{2}{|c|}{$\begin{array}{l}\text { Reference real } \\
\text { strain CAPRI }\end{array}$} & \multicolumn{2}{|c|}{$\begin{array}{l}\text { Transgenic } \\
\text { CAPRI strain }\end{array}$} & \multicolumn{2}{|c|}{ JCVI-Syn 1.0} & \multicolumn{2}{|c|}{ JCVI-Syn 3.0} & \multicolumn{2}{|c|}{ JCVI-Syn3A } \\
\hline 3 & $\begin{array}{l}425653 \\
418009\end{array}$ & 3 & $\begin{array}{l}431863 \\
424453\end{array}$ & 3 & $\begin{array}{l}389604 \\
383147\end{array}$ & 3 & $\begin{array}{l}386328 \\
380244\end{array}$ & 3 & $\begin{array}{l}191148 \\
188330\end{array}$ & 3 & $\begin{array}{l}195794 \\
192178\end{array}$ \\
\hline \multicolumn{2}{|c|}{1.018286688} & \multicolumn{2}{|c|}{1.017457763} & \multicolumn{2}{|c|}{1.01685254} & \multicolumn{2}{|c|}{1.016000252} & \multicolumn{2}{|c|}{1.014963097} & \multicolumn{2}{|c|}{1.018815889} \\
\hline 5 & $\begin{array}{l}349081 \\
342772\end{array}$ & 5 & $\begin{array}{l}353999 \\
348030\end{array}$ & 5 & $\begin{array}{l}322151 \\
314644\end{array}$ & 5 & $\begin{array}{l}319643 \\
312321\end{array}$ & 5 & $\begin{array}{l}158678 \\
155189\end{array}$ & 5 & $\begin{array}{l}162644 \\
158262\end{array}$ \\
\hline \multicolumn{2}{|c|}{1.018405821} & \multicolumn{2}{|c|}{1.01715082} & \multicolumn{2}{|c|}{1.02385871} & \multicolumn{2}{|c|}{1.023443829} & \multicolumn{2}{|c|}{1.022482264} & \multicolumn{2}{|c|}{1.027688264} \\
\hline 8 & $\begin{array}{l}249100 \\
241395\end{array}$ & 8 & $\begin{array}{l}252787 \\
244971\end{array}$ & 8 & $\begin{array}{l}228917 \\
220387\end{array}$ & 8 & $\begin{array}{l}227112 \\
218687\end{array}$ & 8 & $\begin{array}{l}112973 \\
108400\end{array}$ & 8 & $\begin{array}{l}116120 \\
110250\end{array}$ \\
\hline \multicolumn{2}{|c|}{1.03191864} & \multicolumn{2}{|c|}{1.031905817} & \multicolumn{2}{|c|}{1.038704642} & \multicolumn{2}{|c|}{1.038525381} & \multicolumn{2}{|c|}{1.042186347} & \multicolumn{2}{|c|}{1.05324263} \\
\hline 13 & $\begin{array}{l}182285 \\
173428\end{array}$ & 13 & $\begin{array}{l}184673 \\
175894\end{array}$ & 13 & $\begin{array}{l}167319 \\
158110\end{array}$ & 13 & $\begin{array}{l}166119 \\
156734\end{array}$ & 13 & $\begin{array}{l}83100 \\
77329\end{array}$ & 13 & $\begin{array}{l}85559 \\
78424\end{array}$ \\
\hline \multicolumn{2}{|c|}{1.051070185} & \multicolumn{2}{|c|}{1.049910742} & \multicolumn{2}{|c|}{1.05824426} & \multicolumn{2}{|c|}{1.05987852} & \multicolumn{2}{|c|}{1.074629182} & 1.09 & 79802 \\
\hline 21 & $\begin{array}{l}122345 \\
114349\end{array}$ & 21 & $\begin{array}{l}123850 \\
116312\end{array}$ & 21 & $\begin{array}{l}111834 \\
103016\end{array}$ & 21 & $\begin{array}{l}110910 \\
101748\end{array}$ & 21 & $\begin{array}{l}55296 \\
50364\end{array}$ & 21 & $\begin{array}{l}57103 \\
50837\end{array}$ \\
\hline 1.0 & 6278 & 1.06 & 8446 & 1.08 & 8354 & 1.0 & 45996 & 1.09 & 7091 & & 6683 \\
\hline 34 & $\begin{array}{l}80074 \\
73025\end{array}$ & 34 & $\begin{array}{l}81099 \\
74515\end{array}$ & 34 & $\begin{array}{l}72890 \\
66214\end{array}$ & 34 & $\begin{array}{l}72247 \\
65356\end{array}$ & 34 & $\begin{array}{l}36064 \\
31989\end{array}$ & 34 & $\begin{array}{l}37460 \\
32353\end{array}$ \\
\hline 1.0 & 8586 & 1.08 & 8049 & 1.10 & 599 & 1.10 & 7909 & 1.12 & 7539 & 1.15 & 244 \\
\hline 55 & $\begin{array}{l}49907 \\
44879\end{array}$ & 55 & $\begin{array}{l}50665 \\
46176\end{array}$ & 55 & $\begin{array}{l}45287 \\
40085\end{array}$ & 55 & $\begin{array}{l}44848 \\
39272\end{array}$ & 55 & $\begin{array}{l}22000 \\
19301\end{array}$ & 55 & $\begin{array}{l}22992 \\
19368\end{array}$ \\
\hline 1.11 & 34582 & 1.09 & 5003 & 1.12 & & 1.14 & 34111 & 1.13 & 7314 & 1.18 & 2763 \\
\hline 89 & $\begin{array}{l}30152 \\
26319\end{array}$ & 89 & $\begin{array}{l}30708 \\
27108\end{array}$ & 89 & $\begin{array}{l}27153 \\
22722\end{array}$ & 89 & $\begin{array}{l}26742 \\
22243\end{array}$ & 89 & $\begin{array}{l}13030 \\
10741\end{array}$ & 89 & $\begin{array}{l}13749 \\
10743\end{array}$ \\
\hline 1.14 & 36232 & 1.13 & 2125 & $1.1 \mathrm{C}$ & 9242 & 1.20 & 55881 & 1.21 & 8649 & 1.27 & 0109 \\
\hline
\end{tabular}

Comparing Fibonacci TCiAG from 6 mycoplasma genomes dispers ion of values arond ' 1 '

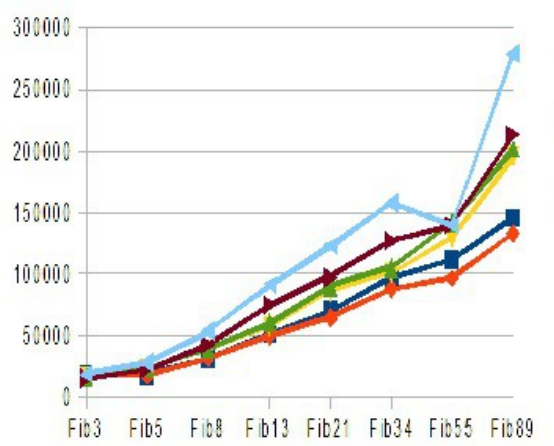

Fibonacci TCIAG from 6 mycoplasma genomes comparing distances regular (forwardi vs reverse (backward)

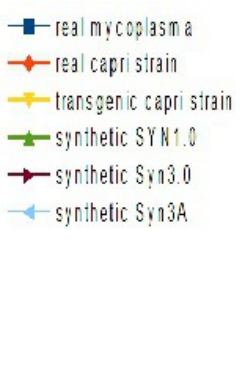

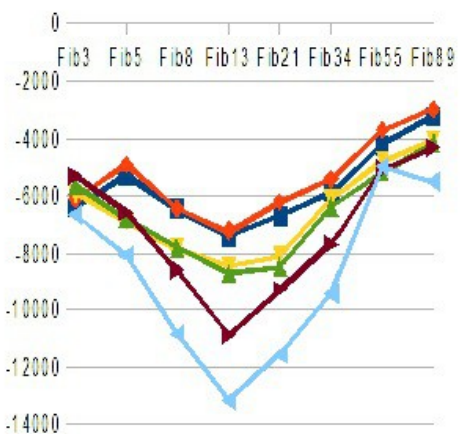

- distance real mycoplasma $\rightarrow$ distance real capri strain $\rightarrow$ distance irans genic capri sirain $\rightarrow$ distance SPH1 $\rightarrow$ istance sin 3.0 $-d i s t a n c e s+13 \mathrm{~A}$

Figure 3 - Left : Comparing TC/AG Fibonacci ratios from 6 mycoplasma genomes (relative values around 1), 
right:Comparing TC/AG Fibonacci distances from 6 mycoplasma genomes. ( regular-reverse distances weighted by the length of the genome, see detailed data in suplementary materials ).

In summary of this double analysis it seems obvious that synthetic genomes disturb and destroy a characteristic dimension of real genomes. This property could concern the mathematical topology of the genome (Rapoport, 2018) and probably its fractal, dynamic, evolution, and three-dimensional structures.

\section{Discussion :}

\section{Comparing real E COLI Genome and synthetic changing TAG by TAA stop codons :}

In (Fredens et al., 2019), researchers published a synthetic genome of E COLI changing systematically genetic code equivalent codons. They replaced every occurrence of the serine codon TCG with AGC, every TCA (also serine amino acid) with AGT, and every TAG (stop codon) with TAA, for a total of 18,214 replacements. Here we run a sample comparison of TG Fibonacci resonances changing stop codons TAG in TAA, then 7725 changes considering only TAG of the first codons reading frame ; In (Fredens et al., 2019), the sequences and genome design details used in this study are available in the Supplementary Data. Supplementary Data 1 provides the GenBank file of the E. coli MDS42 genome (NCBI accession number AP012306.1); Fredens's team systematically replaced every occurrence of the serine codon TCG with AGC, every TCA (also serine) with AGT, and every TAG (stop codon) with TAA, for a total of 18,214 replacements; Not having access to the modified sequence of the synthetic genome yet, we simply changed all TAG codons to TAA codons, that is, 7,725 altered codons. We have limited this change to only the first reading frame codons.

Table 4 - Comparing Fibonacci TG/AC from E-COLI real genome and E-Coli synthetic where all TAG codons are removed in TAA codons ( $1^{\text {st }}$ codons reading frame only) :

\begin{tabular}{|c|c|c|c|}
\hline \multicolumn{2}{|c|}{ ECOLI reference wild type genome } & \multicolumn{2}{|c|}{ ECOLI syn61 like where $7725 \mathrm{TAG}==>$ TAA } \\
\hline 3 & $\begin{array}{l}1471002 \quad 1476399 \\
0.9963444841\end{array}$ & 3 & $\begin{array}{l}1458201 \quad 1484100 \\
0.9825490196\end{array}$ \\
\hline 5 & $\begin{array}{l}1211718 \quad 1215554 \\
0.9968442373\end{array}$ & 5 & $\begin{array}{l}1204279 \quad 1221598 \\
0.9858226683\end{array}$ \\
\hline 8 & $\begin{array}{l}852126 \quad 857586 \\
0.9936332916\end{array}$ & 8 & $\begin{array}{l}844110 \quad 865264 \\
0.9755519703\end{array}$ \\
\hline 13 & $\begin{array}{l}612152 \quad 618151 \\
0.9902952515\end{array}$ & 13 & $\begin{array}{l}604631 \quad 625851 \\
0.9660941662\end{array}$ \\
\hline 21 & $\begin{array}{l}385231 \quad 390203 \\
0.9872579145\end{array}$ & 21 & $\begin{array}{l}378106 \quad 397617 \\
0.9509301665\end{array}$ \\
\hline 34 & $\begin{array}{l}222919 \quad 227478 \\
0.9799585015\end{array}$ & 34 & $\begin{array}{l}216935 \quad 233775 \\
0.9279649235\end{array}$ \\
\hline 55 & $\begin{array}{l}107343 \quad 110152 \\
0.9744988743\end{array}$ & 55 & $\begin{array}{l}102252 \quad 114831 \\
0.8904564099\end{array}$ \\
\hline 89 & 43863451990.9704418239 & 89 & 41531479290.8665108807 \\
\hline
\end{tabular}

Note, the TAG ==> TAA mutations (where $G$ is mutated to A) does not affect the TC / AG structures, we have here to analyze the TG / AC structures. 


\section{E COLIN Natural genome and synthetic changing TAG in TAA stop codons}

Fibonacci TGCCA supracode ratio regular (írward) i reverse (backward!

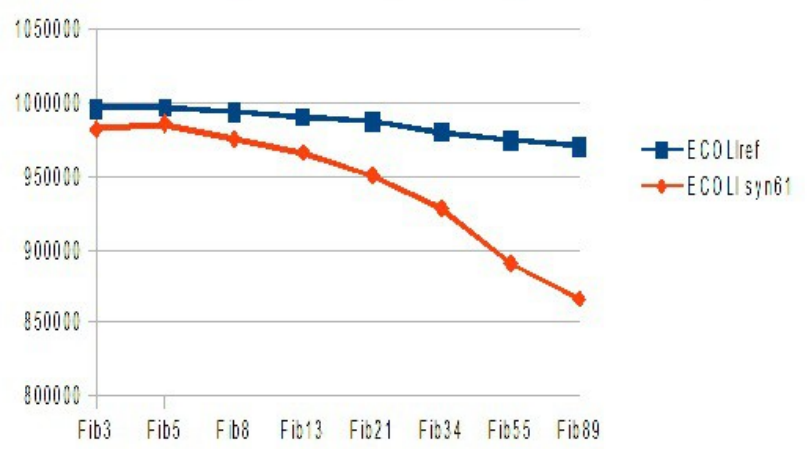

E COLI natural genome and Synthetic changing TAG in TAA stop codons

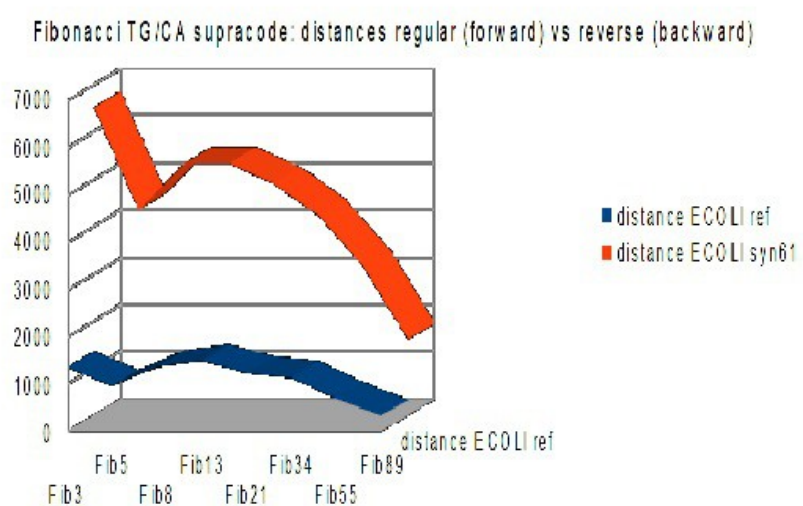

Figure 4 - Left: Comparing TG/AC Fibonacci ratios in real and synthetic E-Coli genomes, Right: Comparing TG/AC Fibonacci distances in real and synthetic E-Coli genomes ( regular-reverse distances weighted by the length of the genome, see detailed data in suplementary materials ).

\section{YEAST SYNTHETIC GENOME, the case of the longer chromosome XII :}

Since 2012 the Synthetic Yeast Genome Project (Sc2.0 http://syntheticyeast.org/sc2-0/ ) results from a worlwide partnership, "Sc2.0 International Consortium team », members spanning 4 continents to provide remote mentorship and solve challenges associated with synthetic individual chromosomes design features and assembly (Jee Loon Foo, 2018).

\section{Sources:}

synthetic yeast project

http://syntheticyeast.org/

\section{7 chromosomes now synthetised} http://syntheticyeast.org/sc2-0-data/

Consorsium has successfully synthesized seven chromosomes. Check the following links to learn about details related to each finished chromosomes:

- synII synIII synV synVI synIXR synX synXII

In (Weiming Zhang et al., 2017) process building the whole synthetic chromosome XII.

Having not yet obtained the synthetic genome from the authors, we have limited here our study to the concatenation of all wild type PCRTags on the one hand and synthetic ones on the other hand. For example:

Forward wild type PCRTag : TGCTTGAACTGCAAATACAGGCCCACTC

Forward synthetic PCRTag : AGCTTGGACAGCGAAAACTGGACCTGAT

They published particularly all the wild type and synthetic PCR Tags.

The full PCR Tags are available online:

\section{http://syntheticyeast.org/wp-content/uploads/2016/10/synXII_PCRtag.txt}

\section{Details : PCRTags}

« PCRTags are alterations incorporated into most open reading frames (ORFs) (on average one per ORF, as some ORFs are too small and others contain multiple PCRTags). These are made by recoding a $\sim 20 \mathrm{bp}$ segments of the coding region of an ORF to a different DNA sequence encoding the same amino acid sequence. PCR primer pairs can then be designed that will selectively 
amplify only the synthetic or wild type sequences. In this way, transformants that have incorporated a synthetic segment can be quickly scanned to ascertain that a complete substitution of the segment has occurred. PCRTags can also be used to monitor for the deletion of non-essential segments post-SCRaMbLE induction. » (from

http://syntheticyeast.org/designs/alterations/pcrtags/ ).

We analysed 681 PCRTags of each $28 \mathrm{bp}$ from wild YEAST XII and artificial SYN XII chromosomes. Then only resonances $<28$ bp are to be considered in the following analysis.

\section{We run 3 analysis :}

Fibonacci sequence $=123581321345589$

Lucas sequence $=13471118294776$

FibLuc sequence= 571219315081131

Table 5 - Comparing real and synthetic YEAST chromosome XII PCRTags with Fibonacci, Lucas and FibLuc resonances:

\begin{tabular}{|c|c|c|c|c|c|c|c|c|c|c|c|}
\hline \multicolumn{6}{|c|}{$\begin{array}{l}\text { YEAST XII real genome } \\
\text { (681 wild type PCRTags) }\end{array}$} & \multicolumn{6}{|c|}{$\begin{array}{c}\text { Synthetic genome SYNXII } \\
\text { (681 synthetic PCRTags) }\end{array}$} \\
\hline \multicolumn{2}{|c|}{ Fibonacci } & \multicolumn{2}{|c|}{ Lucas } & \multicolumn{2}{|c|}{ FibLuc } & \multicolumn{2}{|c|}{ Fibonacci } & \multicolumn{2}{|c|}{ Lucas } & \multicolumn{2}{|c|}{ FibLuc } \\
\hline 3 & $\begin{array}{l}6906 \\
7073\end{array}$ & 3 & $\begin{array}{l}7073 \\
6906\end{array}$ & 5 & $\begin{array}{l}5726 \\
5649\end{array}$ & 3 & $\begin{array}{l}7133 \\
7436\end{array}$ & 3 & $\begin{array}{l}7436 \\
7133\end{array}$ & 5 & $\begin{array}{l}6195 \\
5982\end{array}$ \\
\hline \multicolumn{2}{|c|}{0.9763890853} & \multicolumn{2}{|c|}{1.024181871} & \multicolumn{2}{|c|}{1.013630731} & \multicolumn{2}{|c|}{0.9592522862} & \multicolumn{2}{|c|}{1.04247862} & \multicolumn{2}{|c|}{1.03560682} \\
\hline 5 & $\begin{array}{l}5649 \\
5726\end{array}$ & 4 & $\begin{array}{l}4760 \\
4886\end{array}$ & 7 & $\begin{array}{l}3094 \\
3237\end{array}$ & 5 & $\begin{array}{l}5982 \\
6195\end{array}$ & 4 & $\begin{array}{l}4611 \\
4948\end{array}$ & 7 & $\begin{array}{l}3024 \\
3330\end{array}$ \\
\hline \multicolumn{2}{|c|}{0.9865525672} & \multicolumn{2}{|c|}{0.9742120344} & \multicolumn{2}{|c|}{0.9558232932} & \multicolumn{2}{|c|}{0.9656174334} & \multicolumn{2}{|c|}{0.9318916734} & \multicolumn{2}{|c|}{0.9081081081} \\
\hline 8 & $\begin{array}{l}4012 \\
4124\end{array}$ & 7 & $\begin{array}{l}4894 \\
5032\end{array}$ & 12 & $\begin{array}{l}3518 \\
3552\end{array}$ & 8 & $\begin{array}{l}4142 \\
4396\end{array}$ & 7 & $\begin{array}{l}5272 \\
5456\end{array}$ & 12 & $\begin{array}{l}3636 \\
3918\end{array}$ \\
\hline \multicolumn{2}{|c|}{0.9728419011} & \multicolumn{2}{|c|}{0.9725755167} & \multicolumn{2}{|c|}{0.9904279279} & \multicolumn{2}{|c|}{0.9422202002} & \multicolumn{2}{|c|}{0.9662756598} & \multicolumn{2}{|c|}{0.9280245023} \\
\hline 13 & $\begin{array}{l}2942 \\
3009\end{array}$ & 11 & $\begin{array}{l}2993 \\
3058\end{array}$ & 19 & $\begin{array}{l}1769 \\
1924\end{array}$ & 13 & $\begin{array}{l}2877 \\
3272\end{array}$ & 11 & $\begin{array}{l}2950 \\
3383\end{array}$ & 19 & $\begin{array}{l}1678 \\
2038\end{array}$ \\
\hline \multicolumn{2}{|c|}{0.9777334663} & \multicolumn{2}{|c|}{0.9787442773} & \multicolumn{2}{|c|}{0.9194386694} & \multicolumn{2}{|c|}{0.8792787286} & \multicolumn{2}{|c|}{0.8720070943} & \multicolumn{2}{|c|}{0.8233562316} \\
\hline 21 & $\begin{array}{l}1785 \\
1864\end{array}$ & 18 & $\begin{array}{l}2232 \\
2317\end{array}$ & 31 & $\begin{array}{l}1236 \\
1272\end{array}$ & 21 & $\begin{array}{l}1749 \\
2055\end{array}$ & 18 & $\begin{array}{l}2193 \\
2511\end{array}$ & 31 & $\begin{array}{l}1057 \\
1362\end{array}$ \\
\hline \multicolumn{2}{|c|}{0.9576180258} & \multicolumn{2}{|c|}{0.963314631} & 0.97 & 981132 & 0.8 & 948905 & 0.87 & 72282 & 0.77 & 646109 \\
\hline 34 & $\begin{array}{l}1038 \\
1121\end{array}$ & 29 & $\begin{array}{l}1261 \\
1264\end{array}$ & 50 & $\begin{array}{l}607 \\
623\end{array}$ & 34 & $\begin{array}{l}853 \\
1121\end{array}$ & 29 & $\begin{array}{l}1101 \\
1298\end{array}$ & 50 & $\begin{array}{l}419 \\
566\end{array}$ \\
\hline & 589652 & 0.99 & 265823 & 0.97 & 17817 & 0.7 & 277431 & 0.84 & 80431 & 0.74 & 826855 \\
\hline 55 & $\begin{array}{l}538 \\
507\end{array}$ & 47 & $\begin{array}{l}719 \\
697\end{array}$ & 81 & $\begin{array}{l}221 \\
212\end{array}$ & 55 & $\begin{array}{l}358 \\
438\end{array}$ & 47 & $\begin{array}{l}491 \\
710\end{array}$ & 81 & $\begin{array}{l}172 \\
177\end{array}$ \\
\hline & 43984 & 1.03 & 63845 & 1.04 & 5283 & 0.8 & 515982 & 0.65 & 92958 & 0.97 & 514124 \\
\hline 89 & $\begin{array}{l}195 \\
179\end{array}$ & 76 & $\begin{array}{l}265 \\
237\end{array}$ & 131 & $\begin{array}{l}37 \\
38\end{array}$ & 89 & $\begin{array}{l}105 \\
122\end{array}$ & 76 & $\begin{array}{l}188 \\
212\end{array}$ & 131 & $\begin{array}{l}17 \\
19\end{array}$ \\
\hline 1.08 & 85475 & 1.11 & 4346 & 0.97 & 842105 & $0.8 c$ & 557377 & $0.8 \varepsilon$ & 24528 & 0.89 & 368421 \\
\hline
\end{tabular}


Fibonacci TCIAG Yeast chromosome12

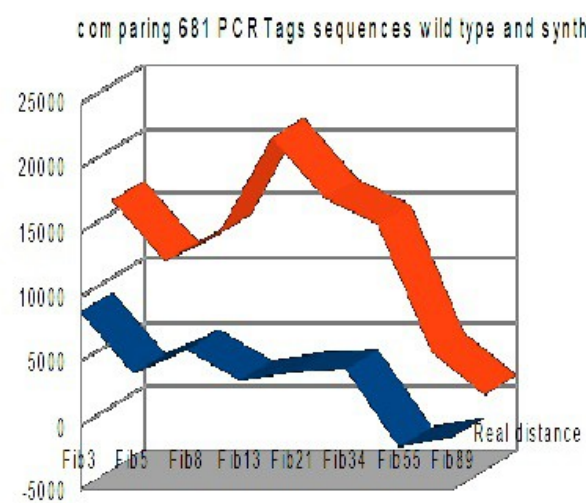

Lucas TCIAG Resonances YEAST chromosome XII

SB1 PCR Tags sequences from wild type and synthetic genomes

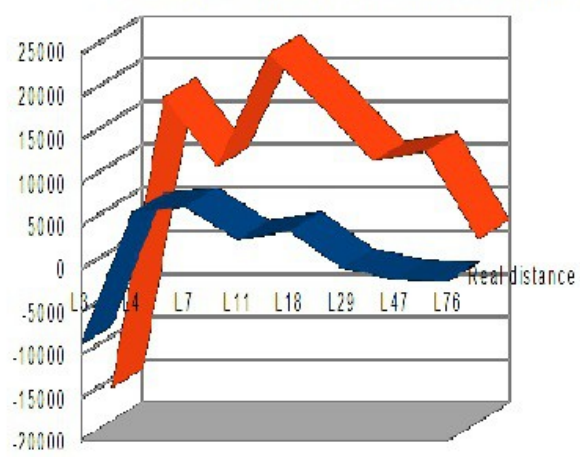

- Real distance

Is sunthetic distance

Fibluc TC/AGResonances YEAST chromosome XII

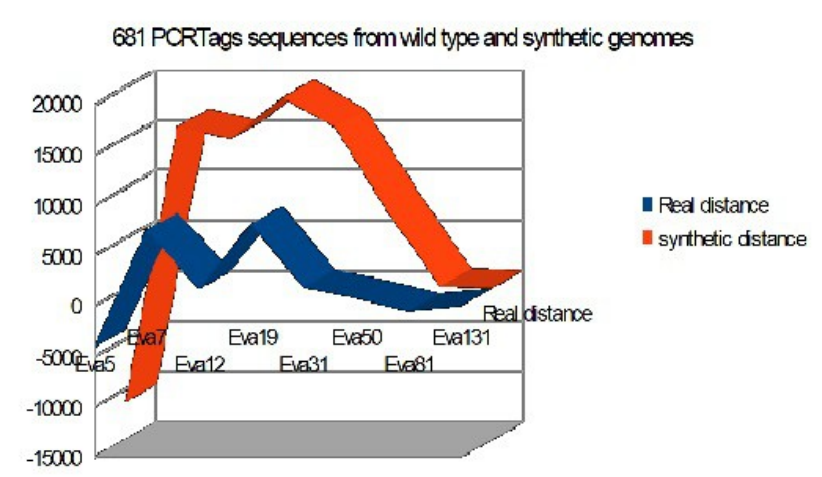

Figure 5 - Comparing TC/AG Fibonacci, Lucas and FibLuc distances in real and synthetic YEAST Chromosome XII PCRTags ( regular-reverse distances weighted by the length of the genome, see detailed data in suplementary materials ).

\section{Conclusion and outlook :}

In all the cases analyzed here, we find that the real genomes or chromosomes have a property of coherence, consistency and unity that our method highlights. This property disappears in almost all (ALL) studied cases of synthetic genomes or chromosomes.

Transposons : a possible explanation of global harmonics structure :

In (Weiming Zhang, 2017), authors write «RATIONALE: The synthetic yeast genome, designated Sc2.0, was designed according to a set of arbitrary rules, including the elimination of transposable elements and incorporation of specific DNA elements to facilitate further genome manipulation.» In our article (Perez, 2010), https://www.ncbi.nlm.nih.gov/m/pubmed/20658335 we wrote : "Why and how could this ancient code be preserved and maintained in spite of the changes and mutations during millions of years of evolution of the human genome?" In the 1940's and 1950's, Nobel prize winner Barbara McClintock discovered a peculiar phenomenon in maize: certain regions of a chromosome moved, or transposed, to other positions. This was the discovery of TRANSPOSONS (Fedoroff, 1984): often called "jumping genes" because of their ability to "jump" to completely different regions within the chromosome and later "jump" back to their original positions. Meanwhile, "jumping genes" is a misleading term because transpositions are related to noncoding areas as well as coding areas. A particular class of transposons moves from one place to another. (Class II transposons consist of DNA sections that move directly from place to place). 
Sometimes there is a palindrome-like swap of the transposon during this move.

Example, the original sequence:

5' TAAGGCTATGC 3'

3'ATTCCGATACG 5'

... Moves to another genome region and becomes

reversed as follows:

5' GCATAGCCTTA 3'

3' CGTATCGGAAT 5'

We found the same process here. It joins a codon with its "mirror-codon". Perhaps DNA double strand topological reshaping processes could explain genesis of the reported facts (hairpin-like unfolding, Moebius-like ribbon, Class II transposons?)... I

These two observations about the role of transposons already partly explain the digital disharmony that we prove in this article. These famous transposons disrupt the functioning of synthetic genomes, so we delete them (!). On the contrary, we believe that these same transposons constitute a major piece of genome stability.

The creation by men of SYNTHETIC genomes leads to a paradox on which I invite you now to think about:

On the one hand, NATURAL DNA is a luxury of REDUNDANCY and SYMMETRY ...

On the other hand, SYNTHETIC DNA manipulation and synthesis technologies rely on and exploit the same luxury of REDUNDANCY and symmetries ... Thus; Sometimes the technology will try to EXPLOIT SYMMETRY and REDUNDANCY: this is the case of CRISPR technology based on DNA PALINDROMES, so on SYMMETRY and REDUNDANCY. Sometimes the technology will try to DESTROY symmetry and REDUNDANCY: Such is the case of mutations and alterations of transposons (Breuer, 2019) in order to fight against these transposons which will alter the SYNTHETIC genome. This is also the case when one tries to reduce the REDUNDANCE of the universal genetic code by reducing it from 64 to 61 codons (Fredens, 2019). By our different research on the biomathematics of DNA, we have on the contrary demonstrated that this REDUNDANCY and this symmetry contribute to the UNITY and INTEGRITY of genes, chromosomes and genomes:

When a Meta-code unifies DNA, RNA and amino acids (Perez, 2009 : Perez, 2011 ; Perez , 2015 ; Perez, 2018d);

When this master code unifies the genomic and proteomic meta structures of a gene (Perez, 2000 ; Perez, 2017e ; Perez, 2017f ; Perez, 2017g ; Perez 2017h);

When the multiple repetition of the same gene as DUF1220 is associated with mammalian brain properties via a kind of «FibLuc sequence » digital standing waves of its DNA (Sikela, 2006 ; Weiss, 2006 ; Parayon, 2011 ; Perez, 2017b);

When we prove the existence of a UNITY of Fibonacci sequences on the scale of an whole human chromosome such as chromosome4 (Perez, 2017c);

When we demonstrate how numerical proportions characterize the DNA of whole genomes of viruses, bacteria or Euchariotes (Perez 2013);

When we highlight the UNITY of the 3 billion base pairs of the entire human genome (Perez, 2010 ; Perez, 2017d);

When this whole human genome UNITY is destroyed by Cancer mutations (Perez, 2018a ; Perez, 2018b ; Perez, 2018c);

When there is an evidence that these numerical structures (Petoukhov, 2019) of the genomes, particularly SYMMETRY and REDUNDANCY, are of TOPOLOGICAL nature (Rapoport 2016). This topological unified hyper structure of whole genomes is based particularly on Fibonacci Numbers, Golden ratio (Friedman, 2018), and Klein bottle (Rapoport§Perez, 2018).

To conclude we will finally notice that the REAL genomes of bacteria analyzed obey two simultaneous numerical constraints of Phi and Phi ${ }^{*} 2$ (where Phi $=1.618$ is the golden 
ratio and $\mathrm{Phi}{ }^{*} 2=2.618$ ). For example, for a contiguous sequence of 21 TCAGs, we have simultaneously:

Regular (forward) 21 TCAG / 8 TC $=\mathrm{Phi}^{*} 2$

And

Reverse (backward) 21 TCAG / 13 TC = Phi.

This double strong constraint on REAL genomes almost disappears in the case of

SYNTHETIC genomes.

We can not manipulate the genomes "no matter how". Thus, transposons certainly play a key role in the stability and epigenetics of genomes.

Manipulation technologies (CRISPR) and especially of artificial creation of genomes will have to respect these laws of nature.

In (Strecker et al., 2019) by using DNA sequences referred to as transposons, or "jumping genes" (genes that can change their position within the genome), a team from MIT led by NYSCF - Robertson Stem Cell Investigator Dr. Feng Zhang has created a new version of CRISPR (called CRISPR-associated transposase, or "CAST") that can insert functional DNA sequences into the genome without making cuts, which can often lead to unintended damage.

\section{Acknowledgements :}

We especially thanks Dr. Robert Friedman M.D. practiced nutritional and preventive medicine in Santa Fe, New Mexico, woldwide expert on Golden ratio Life applications (https://tinyurl.com/y9dxaauv ) and Diego Rapoport (mathématician), Retired Full Professor, UNQ \& Universidad de Buenos Aires, Instituto Balseiro Bariloche; CONICET (Argentina); Universidade de São Paulo \& PUC-Rio (Brasil) ; Univ. Autónoma Metropolitana de México ; Univ. of Tel Aviv ; Univ of Bío Bío (Chile). Patagonia, Argentina. We also thank Marco F. Paya Torres (M.D Alicante), professor E.G. Rajan, Founder President PENTAGRAM Research Centre (P) Limited Hyderabad INDIA, the French biologist Pr. François Gros for its strong comments on HGO and cancer mutations (Pasteur institute,codiscoverer of RNA messenger with James Watson and Walter Gilbert ), Professor Sergey V. Petoukhov (Dr. Phys.-Math. Sci, Grand Ph.D., Full Professor,Laureate of the State prize of the USSR), Volkmar Weiss (Dr. rer. nat. habil. Dr. phil. Habil. Leipzig, Germany), and Pr. Luc Montagnier, medicine Nobel prizewinner for their interest in my research of biomathematical laws of genomes.

\section{References :}

Blount B. A. et al, Construction of an Escherichia coli genome with fewer codons sets records, Nature 569, $492-494$ (2019) doi: 10.1038/d41586-019-01584-x , https://www.nature.com/articles/d41586-019-01584-x

Breuer M. et al., Essential metabolism for a minimal cell., Elife. 2019 Jan 18; 8 Published online 2019 Jan 18. doi: 10.7554/eLife.36842, https://www.ncbi.nlm.nih.gov/pubmed/30657448/

Callaway Ewen, 'Minimal' cell raises stakes in race to harness synthetic life, Nature 531, 557-558 (31 March 2016) doi:10.1038/531557a, https://www.nature.com/news/minimal-cell-raises-stakes-in-race-to-harness-synthetic$\underline{\text { life- } 1.19633}$

Fedoroff, N.V. (1984), Transposable genetic elements in maize. Scientific American 250, 84-98.

Fleaux Rachel, « La musique des genes ». Scientific journal Sciences et avenir , April 1995.

Fraser CM, Gocayne JD, White O, Adams MD, Clayton RA, Fleischmann RD, et al. (1995), The minimal gene complement of mycoplasma genitalium. Science. 1995;270:397-403. [ubMed] [Google Scholar]

https://science.sciencemag.org/content/270/5235/397

Fredens J. et al., Total synthesis of Escherichia coli with a recoded genome, Nature, May 2019, DOI 
https://doi.org/10.1038/s41586-019-1192-5, https://www.nature.com/articles/s41586-019-1192-5

Robert Friedman and Matthew Cross, (2018), Nature's Secret Nutrient: Golden Ratio Biomimicry for PEAK Health, Performance \& Longevity , https://www.amazon.fr/Natures-Secret-Nutrient-BiomimicryPerformance-ebook/dp/B07N8LHDQ1

Hutchinson III et al, Design and synthesis of a minimal bacterial genome, Science 25 Mar 2016: Vol. 351, Issue 6280, aad6253 DOI: 10.1126/science.aad6253; https://science.sciencemag.org/node/676507.full

Gibson DG, Glass JI, Lartigue C, Noskov VN, Chuang RY, Algire MA, et al. (2010), Creation of a bacterial cell controlled by a chemically synthesized genome. Science. 2010;329:52-56. [PubMed] [Google Scholar]

Jee Loon Foo, Synthetic yeast genome reveals its versatility, Nature 557, 647-648 (2018), doi: 10.1038/d41586-01805164-3 https://www.nature.com/articles/d41586-018-05164-3/

Marcer P., Communications: Order and Chaos in DNA — the Denis Guichard Prizewinner: Jean-Claude Pérez, Kybernetes, Vol. 21, 1992, Issue: 2, pp.60 61, https://doi.org/10.1108/eb005922.

Orsini M. et al, Whole-Genome Sequencing of Mycoplasma mycoides subsp. mycoides Italian Strain 57/13, the Causative Agent of Contagious Bovine Pleuropneumonia.

Genome Announc. 2015 Mar 26;3(2). pii: e00197-15. doi: 10.1128/genomeA.00197-15. https://www.ncbi.nlm.nih.gov/pubmed?LinkName=nuccore pubmed\&from uid=731474337

Parayon Gabriel, (2011), On musical self-similarity, ISBN 978-952-10-6970-3 (PDF) : https://helda.helsinki.fi/bitstream/handle/10138/26236/onmusica.pdf

Pellionisz AJ, Graham R, Pellionisz PA, Pérez JC., Recursive Genome Function of the Cerebellum: Geometric Unification of Neuroscience and Genomics. In: Manto M, DL, et al. (Eds) Handbook of the Cerebellum and Cerebellar Disorders. 1st (Edn), Springer, USA, 2012.

Pérez J.C., De nouvelles voies vers I'Intelligence Artificielle - 1988 - Ed. MASSON Paris - ISBN: 2-22581326-4

Pérez JC (1989). De nouvelles voies vers I'Intelligence Artificielle. Ed. Masson Paris Second edition, ISBN: 2-225-81815-0

Pérez JC (1990a). La revolution des ordinateurs neuronaux. Hermes Ed. Paris, ISBN: 2-86601 -203-8. https://www.amazon.fr/R\%C3\%A9volution -ordinateurs-neuronaux-Jean-ClaudePérez/dp/2866012038

Pérez JC (1990b). Integer neural network systems (1.N.N.S) using resonance properties of a Fibonacci chaotic Golden Neuron, Published in: 1990 IJCNN International Joint Conference on Neural Networks , INSPEC Accession Number: 3926657 DOI: 10.1109/IJCNN.1990.137678 Publisher: IEEE Conference Location: San Diego, CA, USA, USA, https://ieeexplore.ieee.org/document/57266 38/

Pérez JC (1990c) Digital Holograms Computers: concepts and apllications. International conference on Neural Networks - LYON France March 1990 ; Springer Verlag. ISBN2-287-0005ISBN2- 287-00051-8 1-8

Pérez JC, Chaos DNA and neurocomputers.

https://www.researchgate.net/publication/258439719_JC_Pérez_1991_Chaos_DNA_and_Neurocomputers_ A_Golden_Link_in_Speculations_in_Science_and_Technōogyvō_14_no_4_ISSN_0̄155-7785

Perez j.C, (1997), « L'ADN décrypté » (Decyphered DNA), Resurgence publisher Liege, Belgium. https://www.amazon.co.uk/LADN-deciphered-security-language-experts/dp/2872110178/ref=asap_bc? ie=UTF8).

Pérez JC (2000). From Prions and Prions-like invariants to the self-assembly thesis. International symposium on Prion Diseases and Elated Processes. Annecy, France.

Pérez JC (2009) Codex Biogenesis. Resurgence, Liege Belgium. https://www.amazon.fr/CodexBiogenesis13-codes-IADN/dp/2874340448 
Perez J.C., Codon populations in single-stranded whole human genome DNA Are fractal and fine-tuned by the Golden Ratio 1.618., Interdiscip Sci. 2010 Sep;2(3):228-40. doi: 10.1007/s12539-010-0022-0. Epub 2010 Jul 25. , https://www.ncbi.nlm.nih.gov/m/pubmed/20658335/

Pérez JC (2011), BIT Life Sciences' 3rd World Congress of Vaccine, Beijing, China" Decoding non-coding Dna Codes:Human Genome MetaChromosomes Architecture " http://fr.scribd.com/doc/57828784/jcPérezB eijing032011

Pérez JC, Applied Mathematics, The "3 Genomic Numbers" Discovery: How Our Genome Single-Stranded DNA Sequence Is "Self-Designed" as a Numerical WholeVol.4 No.10B(2013), Article ID:37457,17 DOI:10.4236/am.2013.410A2004. http://fr.scribd.com/doc/57828784/jcPérezB eijing032011

Perez JC (2015). Deciphering Hidden DNA Meta-Codes -The Great Unification \& Master Code of Biology. J

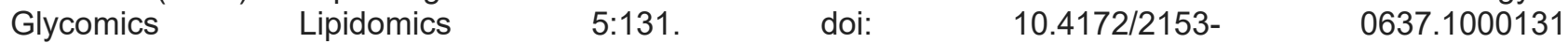
https://www.omicsonline.org/openaccess/deciphering-hidden-dna-metacodesthe-great-unification-mastercode-ofbiology-2153-0637- 1000131.php?aid=55261

Pérez JC (2017a). Sapiens Mitochondrial DNA Genome Circular Long Range Numerical Meta Structures are Highly Correlated with Cancers and Genetic Diseases mtDNA Mutations. J Cancer Sci Ther 2017, 9:512527. doi: 10.4172/1948-5956.1000469, https://www.omicsonline.org/openaccess/sapiens-mitochondrial-dnagenomecircular-long-range-numericalmetastructures-are-highly-correlated-withcancers-and-genetic-disea1948-5956- 1000469. php?aid=90737

Pérez JC (2017b). DUF1220 Homo Sapiens and Neanderthal fractal periods architectures breakthrough(2017)SDRP Journal of Cellular and Molecular Physiology https://www.siftdesk.org/articledetails/DUF1220-Homo-Sapiens-andNeanderthal--fractal-periodsarchitecturesbreakthrough/184

Pérez JC (2017c). The exceptional fractal meta organizations of whole chromosomes 4 of Sapiens, Neanderthal and superior primates, Bioinformatics and Computational BiologyLetters, 2017, 1 (1): 1-26, DOI: 10.24896/bcbl.2017112 https://bacbl.com/index.php/bcbl/article/vie w/2/2

Pérez J C (2017d). A proof of the unity, integrity and autopoietic autonomy of the whole human genome . Research in Biological Chemistry 2017, vol1,no. 1, https://www.rbchemistry.com/index.php/rb c/article/view/2

Pérez JC (2017e). The Master Code of Biology: from Prions and Prions-like Invariants to the Self-assembly Thesis. Biomed J Sci\&Tech Res1(4)- 2017. BJSTR.MS.ID.000369. DOI: 10.26717/BJSTR.2017.01.000369 https://biomedres.us/pdfs/BJSTR.MS.ID.00 0369.pdf

Pérez JC (2017f). Decyphering "the MASTER CODE ®" Structure and Discovery of a Periodic Invariant Unifying 160 HIV1/HIV2/SIV Isolates Genomes. Biomed J Sci \& Tech Res 1(2)-2017. BJSTR. MS.ID.000209. http://biomedres.us/pdfs/BJSTR.MS.ID.000 209.pdf

Pérez JC (2017g). The "Master Code of DNA: Towards the Discovery of the SNPs Function (SingleNucleotide Polymorphism). J Clin Epigenet. Vol. 3 No. 3:26, http://clinicalepigenetics.imedpub.com/themaster-codeof-dna-towards-the-discovery-of-the-snpsfunction-singlenucleotidepolymorphism.pdf Pérez JC (2017e), Mannheim Germany 27 November 2017 Biomathematics conference in Mannheim Germany https://www cammbio.hsmannheim de/kick-off-event html https://www.cammbio.hsmannheim.de/fileadmin/user_upload/projek te/cammbio/events/20171127kickoff/EPPérez.pdf

Pérez JC (2017h). The Master Code of Biology: Self-assembly of two identical Peptides beta A4 1-43 Amyloid In Alzheimer's Diseases. Biomed J Sci \& Tech Res 1(4)- 2017. BJSTR.MS.ID.000394. DOI: 10.26717/BJSTR.2017.01.000394, https://biomedres.us/pdfs/BJSTR.MS.ID.00 0394.pdf

Pérez JC (2018a). Towards a Universal Law Controlling all Human Cancer Chromosome LOH Deletions, Perspectives in Prostate and Breast Cancers screening. Canc Therapy \& Oncol Int J. 2018; 9(2): 555756. DOI: 10.19080/CTOIJ.2018.09.555756. https://juniperpublishers.com/ctoij/pdf/CTO IJ.MS.ID.555756.pdf

Pérez JC (2018b). Cancer, Quantum Computing and TP53 Tumor Suppressor Gene Mutations Prediction. Nov Appro in Can Study. 1(2). NACS.000507.2018. http://crimsonpublishers.com/nacs/pdf/NA CS.000507.pdf 
Pérez JC (2018c). Neuroblastoma and Glioblastoma Brain Cancers: «Human Genome Optimum» (HGO) a Global Genome Strategy controlling all Human Chromosome LOH Deletions. Nov Appro in Can Study. 1(3). NACS.000512.2018.http://crimsonpublishe rs.com/nacs/pdf/NACS.000512.pdf

Pérez, J.C. (2018d) .Six Fractal Codes of Biological Life:perspectives in Exobiology, Cancers Basic Research and Artificial Intelligence Biomimetism Decisions Making. Preprints 2018, 2018090139 (doi: 10.20944/preprints201809.0139.v1).http:// www.preprints.org/manuscript/201809.013 9/v1

Perez J.C. (2019), 20219 paper to be published, "The Discovery of a "DNA SUPRACODE" Controlling Locally \& Globally Humans Mitochondrial Genomes: Perspectives in Evolution and Cancers Early stage Diagnostic ».

Sergey V. Petoukhov, Zhengbing Hu, Matthew He, Advances in Artificial Systems for Medicine and Education II, DOlhttps://doi.org/10.1007/978-3-030-12082-5, Springer Nature Switzerland AG 2020 , https://link.springer.com/book/10.1007\%2F978-3-030-12082-5\#page=40

Rapoport D.L., Klein Bottle Logophysics, Self-reference, Heterarchies, Genomic Topologies, Harmonics and Evolution. Part III: The Klein Bottle Logic of Genomics and its Dynamics, Quantum Information, Complexity and Palindromic Repeats in Evolution Quantum Biosystems | November 2016 | Vol 7 | Issue 1 | Page 107174107 ISSN 1970-223X www.quantumbiosystems.org

Rapoport D. L. and Perez J.C. (2018) Golden ratio and Klein bottle Logophysics: the Keys of the Codes of Life and Cognition. Quantum Biosystems. 9(2) 8-76.Vol. 9- n.2 - 2018

Sikela, J. M., The jewels of our genome: the search for the genomic changes underlying the evolutionarily unique capacities of the human brain. PLoS Genetics 2 (2006) doi.org/10.1371/journal.pgen.0020080

Sleator RD., The story of Mycoplasma mycoides JCVI-syn1.0: the forty million dollar microbe. Bioeng Bugs. 2010;1(4):229-230. doi:10.4161/bbug.1.4.12465

Strecker J, Ladha A, Gardner Z, Schmid-Burgk JL, Makarova KS, Koonin EV, Zhang F., RNA-guided DNA insertion with CRISPR-associated transposases., Science. 2019 Jun 6. doi: 10.1126/science.aax9181. https://doi.org/10.1126/science.aax9181

Venetz JE et al, Chemical synthesis rewriting of a bacterial genome to achieve design flexibility and biological functionality, PNAS 2019, doi: $\underline{10.1073 / p n a s .1818259116}$

Weiming Zhang et al., Engineering the ribosomal DNA in a megabase synthetic chromosome Science 10 Mar 2017: Vol. 355, Issue 6329, eaaf3981 DOI: 10.1126/science.aaf3981

Weiss $\mathrm{H}$ and Weiss V., The golden mean as clock cycle of brain waves - Chaos, Solitons \& Fractals, 2003 